\title{
VORTICITY DYNAMICS IN THREE-DIMENSIONAL PULSATING CO-FLOWING JET DIFFUSION FLAMES
}

\author{
JUAN C. LASHERAS \\ Department of Applied Mechanics and Engineering Sciences \\ University of California, San Diego \\ La Jolla, CA 92093 USA \\ AND \\ AMABLE LIÑÁN, ANTONIO LECUONA AND PEDRO RODRIGUEZ \\ E.T.S.I. Aeronáuticos \\ University Politécnica de Madrid. 28040 Madrid, Spain
}

\begin{abstract}
The vorticity dynamics in the near field of laminar $\left(\mathrm{Re}=10^{3}\right)$ co-flowing jets subjected to the single or combined effects of axial and azimuthal forcing is analyzed. It is shown that the interaction of the three-dimensional vortex structure resulting from the growth of the two and three-dimensional instabilities may result in large changes in the entrainment and mixing characteristics of the jet. For each azimuthal forcing, and for a fixed velocity ratio between the inner and outer jet, we show the existence of several instability modes leading to a pattern of lateral ejections of closed vortex loops. These modes and their topological changes are analyzed in view of the three-dimensional inviscid induction of the two concentric array of vortex rings emanating from the jet's exit nozzle. For the case of methane-air diffusion flames, flow visualizations revealed the existence of qualitatively similar patterns of closed flame cells and fingers.
\end{abstract}

\section{Introduction}

The axisymmetric instability of a laminar shear layer of cylindrical shape is well understood, both from an analytical and experimental point of view ${ }^{1,2,3,4,5,6}$ among many others. This instability results in the roll up of the cylindrical vorticity sheet to form a periodic array of vortex rings. This evolution has also been corroborated as occurring in transitional and turbulent jets ${ }^{7,8,9,10,11}$ and others. In essence, these high Reynolds number studies have shown that early in the evolution of a fully developed turbulent jet, the primary vorticity concentrates to form a coherent array of ring-like vortical structures.

Browand and Laufer, ${ }^{7}$ Yule, ${ }^{8}$ and others have shown that the transition to turbulent flow in jets involves a relatively orderly three-dimensional deformation of the initial vortex rings. Their results show that after the growth of the primary instability, the vortex rings further develop a high azimuthal structure whose breakdown may occur in a violent manner. During the breakdown process, they also observed that pieces of the core's vorticity can pinch off to form small vortex loops. Yule ${ }^{8}$ then argued the importance of this azimuthal instability mode of the vortex rings, suggesting that transition in round jets involves the entanglement of the street of coherent vortex rings which have developed along their circumference wave deformations.

The instability of the ring-like vortices observed in jets has further been associated with the shortwave instability found in isolated vortex rings by Widnall and Sullivan, ${ }^{12}$ Widnall, et al., ${ }^{13}$ Maxworthy ${ }^{14,15}$ and others. These analytical and experimental studies have shown that the instability manifests itself in the generation of waves over the circumference of the ring. The thinner the inner core of the vortex ring, the larger is the more unstable number of waves found to be amplified through this ring's instability.

In order to elucidate the mechanisms leading to the three-dimensional transition of the jet, and in particular to study the role that the ring's instability plays in the three-dimensional transition (and eventually in the turbulent transition), we conducted a set of experimental studies in which laminar, coflowing jets were subjected to the combined effect of a pair of orthogonally oriented perturbations. The first one (hereafter referred to as the primary perturbation) consisted of a single wave, sinusoidal perturbation in the axial velocity component of the jet at the nozzle's exit section. The use of this primary perturbation was intended to lock to a given 
value (at least in the near region of the jet) the frequency and thus the wavelength of the jet's vortex rings. The second one (hereafter referred to as the secondary perturbation) consisted of a wavy, sinusoidal radial displacement of the cylindrical vorticity layer emanating from the nozzle. This was achieved by means of a corrugated nozzle exit whereby the cylindrical vorticity layer emanating from it is periodically displaced radially in and out along the jet circumference. Early experimental and numerical work on the effect of this second perturbation ${ }^{16,17,18}$ have shown that the growth and amplification of this secondary perturbation results in the formation of counter-rotating pairs of streamwise vortices which further interact with the vortex rings locking their azimuthal mode to a predetermined number of waves along its circumference. Thus, the intention of the secondary perturbation was to lock the ring's instability to a given azimuthal wavelength (i.e., number of waves along its circumference). The work is, in this respect, an extension of the axisymmetric configuration of similar studies conducted for free shear layers and wakes in the planar configuration ${ }^{19,20,21,22}$ where we analyzed the combined growth of streamwise and spanwise perturbations.

Although our investigation has the general objective of obtaining a clearer understanding of the nature of the three-dimensional transitions which occur in the near region of co-flowing, round jets diffusion flames developing from thin, laminar boundary layers, this paper has a less broad scope. Rather, it is devoted to demonstrating that the structure of the near region of the reacting jet can be altered considerably as a result of the growth and nonlinear interactions between the vortical structures formed through the jet's instabilities. Without intending to provide an exhaustive analysis, we will concentrate only on some cold flow results and its extension to preliminary diffusion flame studies.

\section{The Experimental Set-up and Observation Techniques}

The experiments were conducted in a highly versatile, atmospheric pressure, open-return wind tunnel where non-reacting (isothermal) as well as heat transfer or combustion experiments can be conducted. A detailed description can be found in Lecuona et al. ${ }^{23}$

The results reported here correspond to the case of axial forcing by a single sinusoidal wave with a frequency ranging from 0 to $20 \mathrm{~Hz}$. In addition, we introduced azimuthal perturbations following the same techniques used in early studies of plane, free shear flows 19,20 i.e., corrugated or indented nozzles. The effect of a gradual corrugation at the trail- ing edge of the nozzle is to periodically displace radially in and out the cylindrical vorticity layer emanating from the exit section. In the present case we will restrict the azimuthal perturbation to the case of the corrugated nozzle only. The perturbation amplitude was about $+/-3 \mathrm{~mm}$ in both cases, significantly smaller than the boundary layer thickness of the two streams. Although the number of waves per circumference was varied from four to nine, we will restrict the discussion here to the case of five waves per revolution only.

In order to reveal the three dimensional structure of the flow we used a combination of flow visualization techniques. The inner, primary flow is first dried and subsequently saturated with $\mathrm{TiCl}_{4}$ vapors, meanwhile the secondary flow is supplied containing water vapor in a concentration equal to that existing in the ambient laboratory conditions. As the two streams meet at the edge of the nozzle the $\mathrm{TiCl}_{4}$ vapors react with the water vapor to form submicron size $\mathrm{TiO}_{2}$ particles. The reaction products $\left(\mathrm{TiO}_{2}\right)$ provide a continuous generation of low diffusivity scattering particles that will mark the instantaneous position of the interface.

\section{Three-Dimensional Patterns in the Near Region of the Forced Co-Flowing Jets}

\section{Non Reacting, Co-Flowing Cold Jets:}

The following is a preliminary study intended to provide experimental evidence of the existence of a large variety of entrainment patterns that may result from the combined effect of the axial and azimuthal forcing. The work is exploratory in nature and no attempt will be made here to fully characterize the behavior of the jet for the whole range of parameters, i.e. the jet's Reynolds number, the frequency and amplitude of the axial forcing and the wavelength and amplitude of the azimuthal forcing. In particular, we will discuss only a set of experiments where a co-flowing jet has been strongly forced in the axial and in the azimuthal directions to show the development of several different vortical patterns resulting from changes in the initial conditions. A more detailed and complete parametric study including an exhaustive analysis of all the different three-dimensional vorticity modes and their analysis involving inviscid vortex dynamics simulations will be presented elsewhere ${ }^{24}$ There, we present an exhaustive comparison between experimentally found patterns and inviscid dynamics simulations.

Martin, Meiburg and Lasheras, ${ }^{16}$ using three-dimensional inviscid vortex dynamics simulations, have shown that a smooth, cylindrical vorticity layer when perturbed both with a single sinusoidal wave in the axial direction and an azimuthal, sinusoidal pertur- 


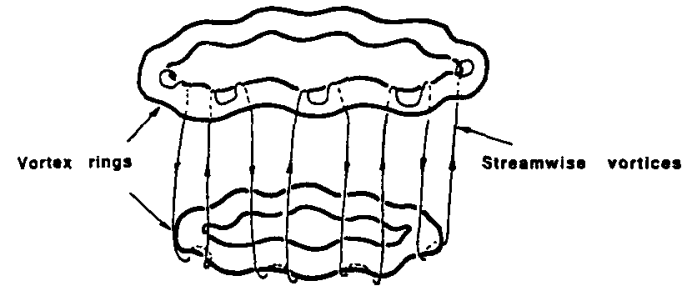

FIG. 1. Vortex patterns resulting from the combined effect of axial and azimuthal perturbations. Note the presence of counter-rotating pairs of streamwise vortices which wrap around two consecutive vortex rings.

bation of a given wavelength results not only in the roll-up of the vorticity layer to form vortex rings, but also in an additional array of streamwise vortices located in the bridges connecting two consecutive rings (Fig. 1). The observed evolution can be summarized in the following sequence of events. A: the growth of the primary, axial instability leads to the periodic concentration of vorticity in vortex rings located in planes perpendicular to the axis of the cylinder. B: Under the effect of the evolving positive strain rate which is created between two consecutive rings, the perturbation vorticity is tilted, reoriented, and stretched to form counter-rotating pairs of vortex tubes that eventually wrap around the rings.

Since in the present study we will be looking at the evolution of strongly forced, co-flowing jets, the global vorticity dynamics may be considerably more complicated than the one described above. However, an extrapolation of Meiburg's and Martin's calculations based on our early studies of three-dimensional plane wakes ${ }^{21,22}$ suggest that co-flowing forced jets should be modelled using two concentric vorticity cylinders of opposite sign with the appropriate relative strength between them given by the properties of the corresponding boundary layers. For large amplitudes of forcing, the effect of the primary perturbations can lead to alternate roll ups of the two vorticity layers so that a staggered array of vortex rings of opposite sign is formed. In addition, the growth and amplification of the secondary (azimuthal) perturbation now results in an array of counter-rotating vortex tubes located in the braids connecting two consecutive vortex rings of opposite sign (much as was the case with plane wakes, Lasheras and Meiburg, ${ }^{21}$ their fig. 10). As was the case in the plane wake, the topology of the resulting set of vortex tubes and rings would depend not only on the amplitude of the axial forcing, but also on the orientation of the azimuthal perturbation (indented or corrugated nozzle).

In general, depending on the amplitude and frequency of the axial forcing and on the boundary layer characteristics, the above described evolution will lead to two opposite sign, coaxial rings per wavelength located in a staggered configuration with a certain relative magnitude and core radius. As a consequence of the interaction with the counter-rotating pairs of streamwise vortices, each of these vortex rings will have wavy dislocations in their cores of the same wavelength as the imposed azimuthal perturbation. However, the wavy dislocation in their cores will be either in phase or out of phase, depending on the orientation of the initial azimuthal perturbation, i.e. a co-flowing jet issuing from a corrugated nozzle when strongly forced, will develop a Karman-like vortex ring pattern with the vortices of each sign containing a circumferential wavy dislocation $180^{\circ}$ out-of-phase between two rings of opposite sign. However, a jet issuing from an indented nozzle would produce a similar pattern, but the staggered array of opposite sign vortex rings would contain circumferential wavy dislocations inphase with each other. The global induction of this highly complicated vorticity organization can subsequently lead to a large variety of topological changes. These topological changes and their effect in the entrainment and spreading of the jet is precisely the point intended to be addressed in this paper.

In the following, we elected to discuss four different modes of three-dimensional patterns observed in laminar, co-flowing jets with Reynolds numbers based on the inner jet's diameter and relative velocity of the order of 1000 . The frequency of the axial forcing was varied between 4 and 20 $\mathrm{Hz}$, and the number of lobes in the corrugated nozzle was 5 .

\section{Mode 1:}

In the case of small amplitude forcing and axial forcing frequencies resulting in wavelengths of the order of the jet diameter, the near field structure of the vorticity was found to evolve along the mechanism described in Fig. 1. i.e., the dynamics can be captured with the inclusion of only one vorticity cylinder as shown by Martin, Meiburg and Lasheras, ${ }^{16}$ and the wake-like effect of the existence of the outer boundary layer can be neglected. A horizontal cross cut of the jet at a downstream distance of three jets' diameters (Fig. 2) reveals the presence of counter-rotating pairs of streamwise vortices as well as the circumferential, wavy undulation in the cores of the vortex rings. This crosscut has been made through the core of the vortex ring. The two concentric interfaces reveal the roll up of the vorticity sheet. A perspective view of the jet's interface corresponding to a jet length spanning between the first and fourth roll up is shown in Fig. 3 where the lambda shape of the streamwise vortices can be clearly seen. At this down- 


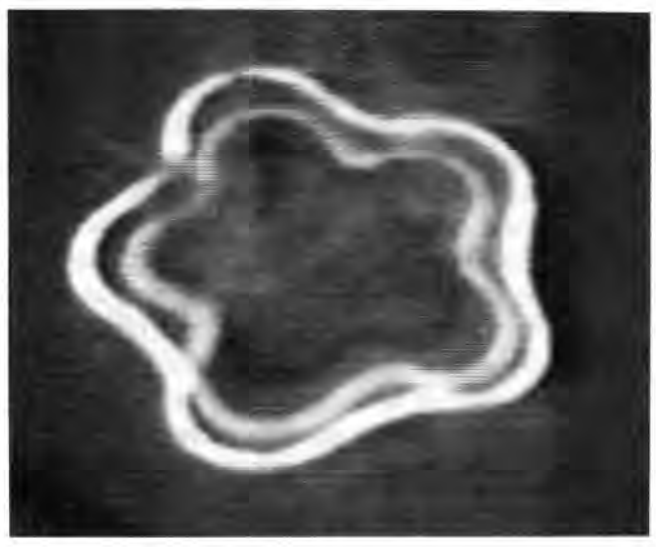

FIG. 2. Mode 1. Horizontal cross-cut of the jet at a downstream distance of 3 jet diameters (Section A-A. in Figure 6.) The interface is marked by the white product (for the purpose of better visualization, we are showing the negative of the recorded image).

stream location, the instability of the vortex ring $^{12,13,14,15}$ has not grown appreciably.

\section{Mode 2:}

For axial forcing frequencies higher than the most unstable one found for the natural case, and for low values of its amplitude, was found to lead to a new

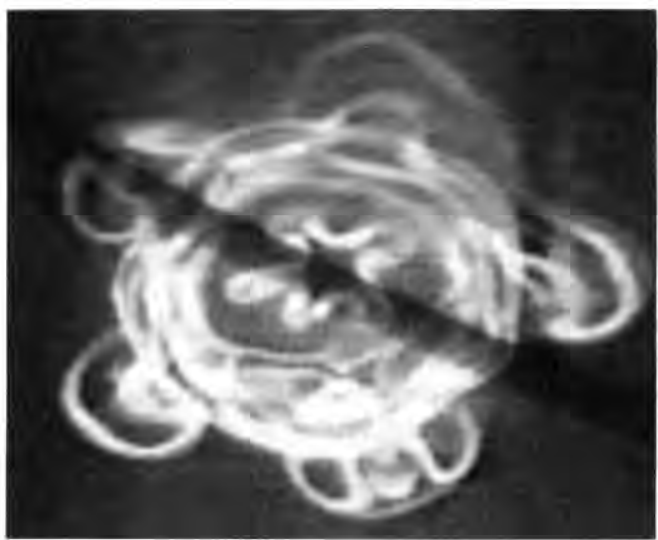

FIG. 3. Mode 2. Horizontal cross-cut of the jet at a downstream distance of 3 jet diameters. Observe the presence of 5 closed vortex loops in the process of being pinched off from the jet. The vortex loops are located aligned with the valleys of the corrugated nozzle. mode of three-dimensional vortex patterns consisting of a symmetric mode of lateral ejections. In Fig. 3 , we show a horizontal cross-cuts of the jet at a downstream distance of three jet diameters. Note the appearance of closed vortex loops forming in the outer portion of the vortex ring which occur at radial locations aligned with the valleys of the corrugated nozzle. For these high levels of the amplitude of the axial forcing, the ring structure of the jet is composed of a staggered array of pairs of vortex rings of opposite sign. Resulting from the amplification of the ring's instability, the outer-most weaker vortex ring is observed to deform to a stage by which vortex loops reconnect and pinch off as schematically shown in Fig, 4. Under their own induction, the vortex loops pinching off from the outer ring, are propelled outwards; thus ejecting chunks of fluid from the jet into the outer stream. In the horizontal cross-cut of Fig. 3, observe that because of the $180^{\circ}$ phase shift between wave perturbation of the inner and outer ring, the azimuthal location of the pinch-off corresponds to the valleys of the corrugated nozzle.

\section{Mode 3:}

For amplitudes of forcing up to $50 \%$ of the initial streamwise velocity, we observe the development of a third, and more dramatic, three-dimensional symmetric mode. A horizontal cross-cut through the ring at a downstream distance of three jet diameters (Fig. 5b) now shows the appearance of closed vortex loops being ejected at circumferential locations coinciding with the crests of the corrugations. A possible mechanism consistent with all our visual data which could be responsible for this new threedimensional vortex pattern is schematically shown in Fig. 6. Note that in this case, the amplification of the perturbation of the instability of the primary, inner ring leads to the pinch-off of stronger vortex loops which are located at azimuthal positions aligned with the crests of the corrugated nozzle. Observe in the horizontal cross-cut of the jet (Fig. 5a) the reconnected inner vortex ring as well as the five strong vortex loops ejecting sideways. Observe also five weaker loops which had resulted from the reconnection of the outer ring also visible in between two of the strong loops. The spreading angle of the jet at the location aligned with the crests of the corrugation (location of the ejection of the strong loops) was found to be considerably enhanced with respect to the observed in the previous two modes, indicating that the self-propelled motion of this new set of vortex loops is much stronger than the ones observed forming from the outer ring.

\section{Mode 4:}

As the axial forcing frequency is further increased above the most unstable one, we found the 

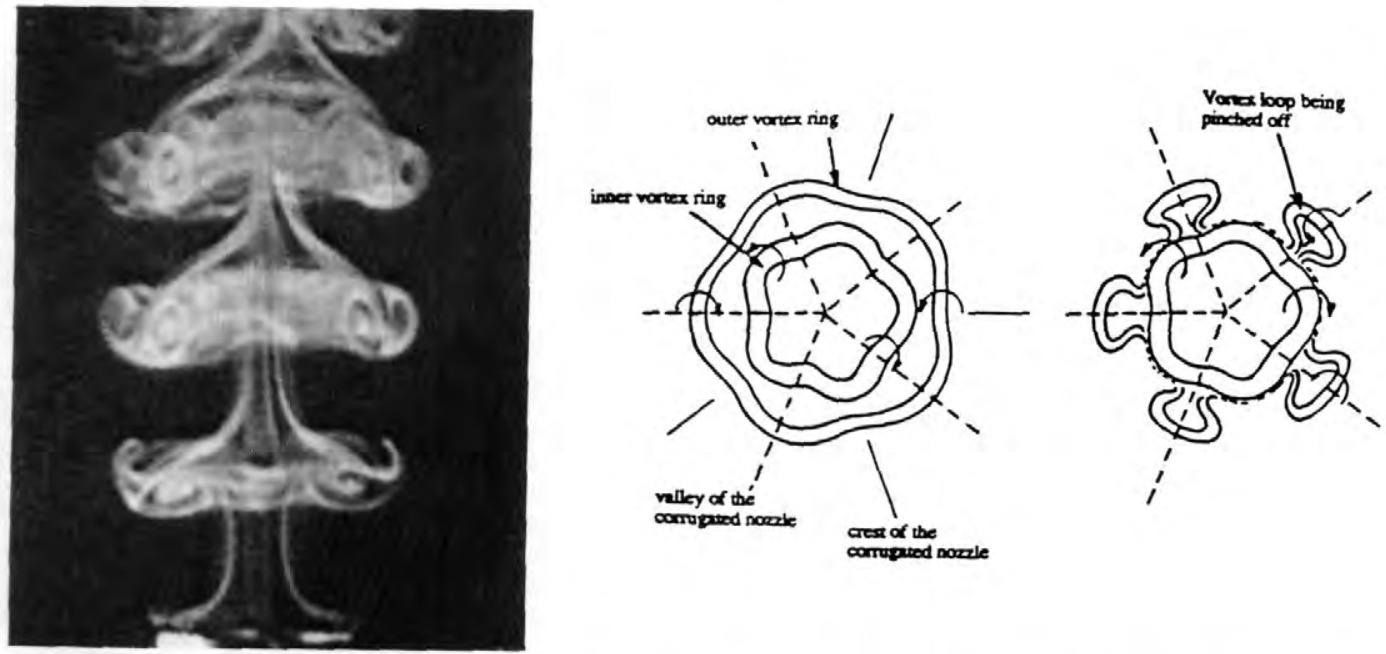

Fig. 4. Mode 2. A. Side-view interface flow visualization. Notice the double roll-up of the vorticity layer to form a staggered array of co-axial vortex rings of opposite sign. Also noticeable are the streamwise vortices present in the stem (braids) connecting two consecutive vortex rings. B. Schematic representation of the combined evolution of the inner and outer ring leading to the pinch-off of closed vortex loops at azimuthal locations aligned with the valleys of the corrugated nozzles. The short wave instability of the outer, weaker ring, under the induction of the stronger inner one, leads to the pinch-off and reconnection of 5 closed vortex loops.
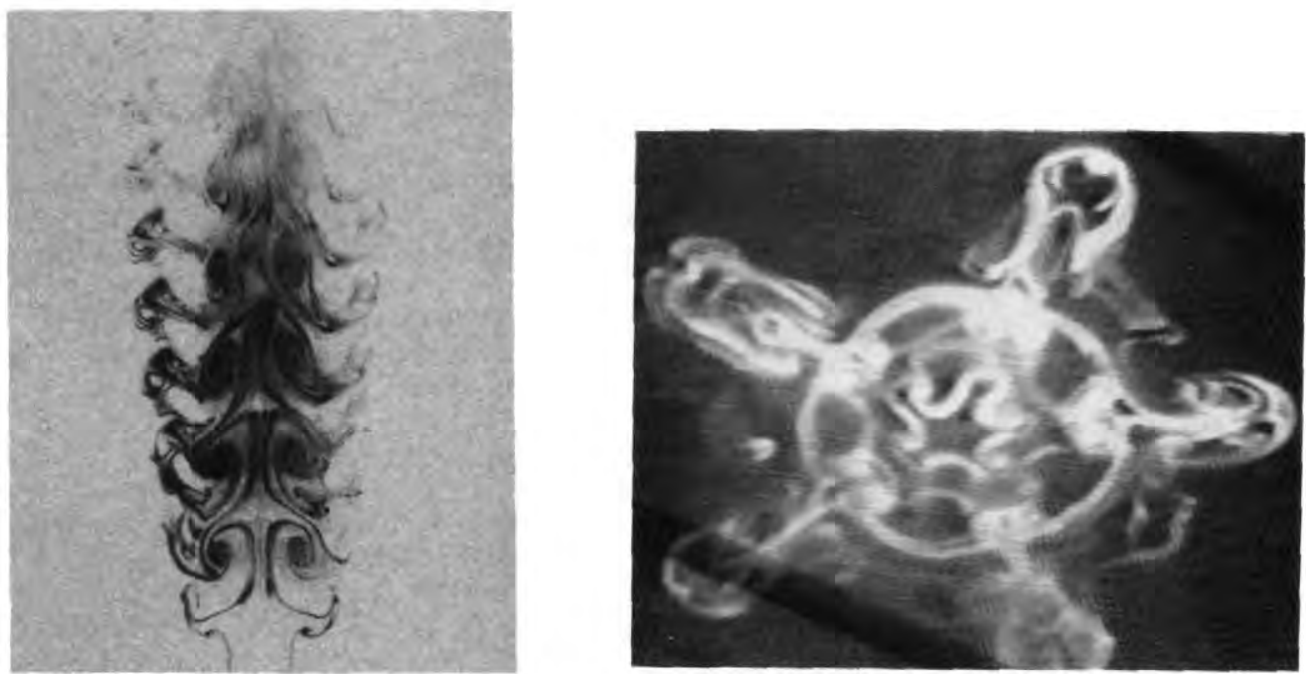

FIG. 5. Mode 3. (a) Vertical cross-cut of the jet (left photograph). (b) Horizontal cross-cut of the jet at a downstream distance of 3 jet diameters (right photograph). Note the existance of 5 closed vortex loops forming at azimuthal locations aligned with the crest of the corrugated nozzle. Observe also the reconnected inner ring after the pinch-off process. 

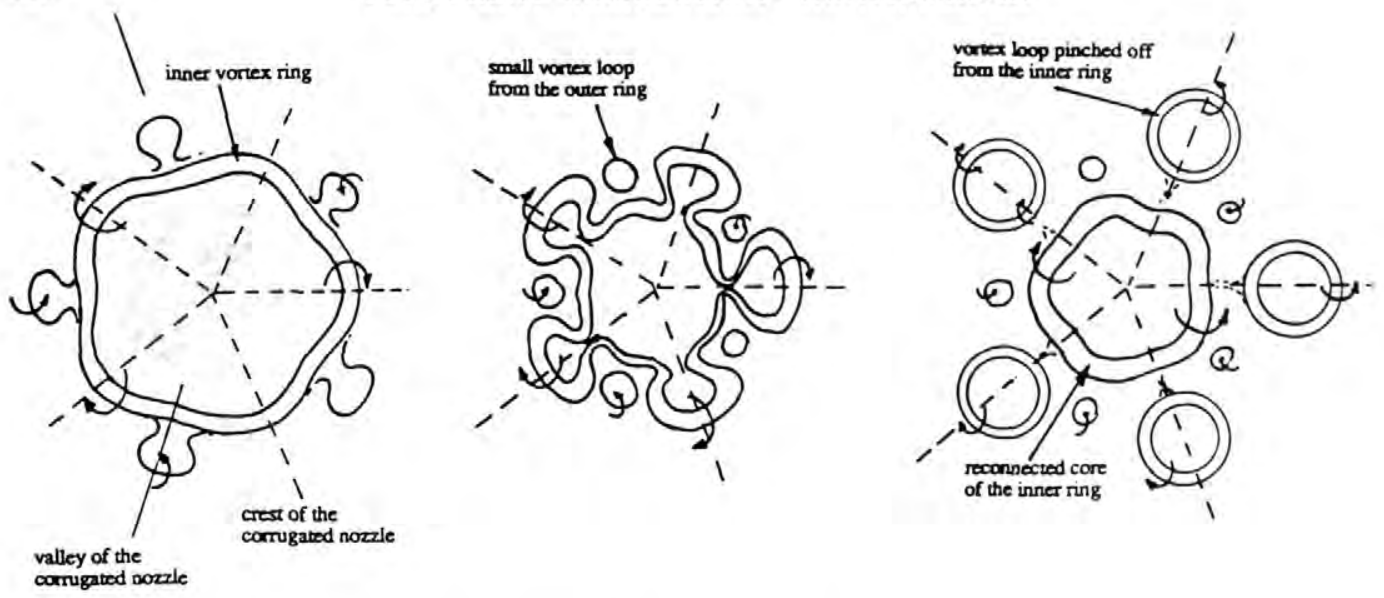

FIG. 6. Mode 3. Schematic representation of the growth and amplification of the short wave instability of the inner vortex ring resulting now in a formation of closed vortex loops pinching off from azimuthal locations aligned with the crests of the corrugated nozzle, as shown in Figure 13.

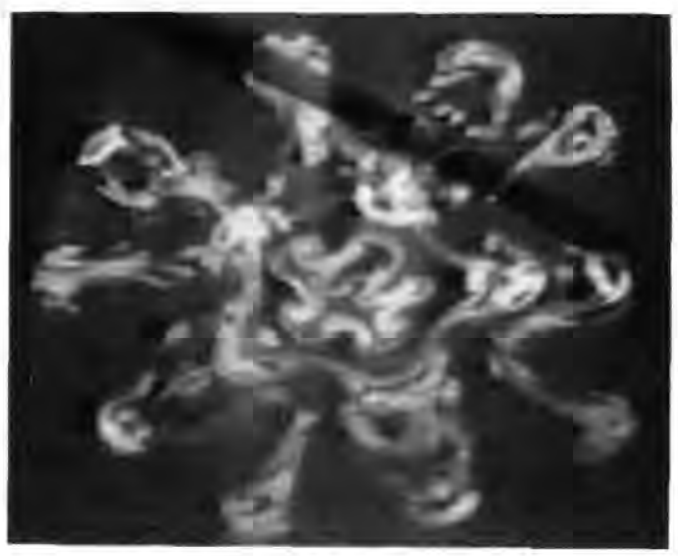

Fig. 7. Mode 4. Horizontal cross-cut at a downstream distance of 3 jet diameters showing the existance of ten vortex loops being ejected (doubling the number of ejections).

appearance of a new three-dimensional symmetric mode composed of a double number of lateral ejections (Fig. 7). This mode appear to be the result of the simultaneous instability of both inner an outer rings.

Although the experiments described here were only concerned with laminar, co-flowing jets, our results conducted with transitional and turbulent coflowing jets confirm the existence of similar mechanisms by which the jets develop three-dimensional patterns of lateral ejections. Thus, it appears that some of our observed mechanisms described above for laminar flows could also be responsible for the lateral ejections reported in transitional and fully developed turbulent jets. ${ }^{22,26,27,28,29}$

\section{Methane-Air Co-Flowing Jets Diffusion Flames:}

In this section we present preliminary results intended to shown that strongly forced co-flowing jets diffusion flames may qualitatively exhibit the formation of the same patterns of the vorticity field encountered in non-reacting jets and described above. The present results are restricted to the case where the inner jet is supplied with a methane/ nitrogen mixture with dilution ratio of 0.3 and the outer jet is air. The inner and outer jet velocities are $1.0 \mathrm{~m} / \mathrm{s}$ and $0.3 \mathrm{~m} / \mathrm{s}$ respectively. These conditions correspond to a Reynolds number of the order of $10^{3}$ and initial Richardson numbers of the order 1 .

Figure 8 shows a direct flow visualization of the flame in the case where the flow was forced only axially at a frequency of $12 \mathrm{~Hz}$ (round nozzle). Note that under the effect of the primary shear instability, the vorticity concentrates to form a periodic array of vortex rings. Under the influence of the velocity field generated by the vortex rings the flame wrap around their cores. In addition observe that in the braids (or bridges) connecting two consecutive vortex rings, the flame is stretched and shows the presence of an array of streamwise vortex pairs similar to those described in Figure 1.

Figure 9 shows the effect of the inclusion of azimuthal forcing. When the flow is simultaneous forced axial and azimuthally, the flame exhibit the presence of vortex rings and counter rotating pairs of axial vortex tubes. Observe that these streamwise vortices when interacting with the vortex ring in- 


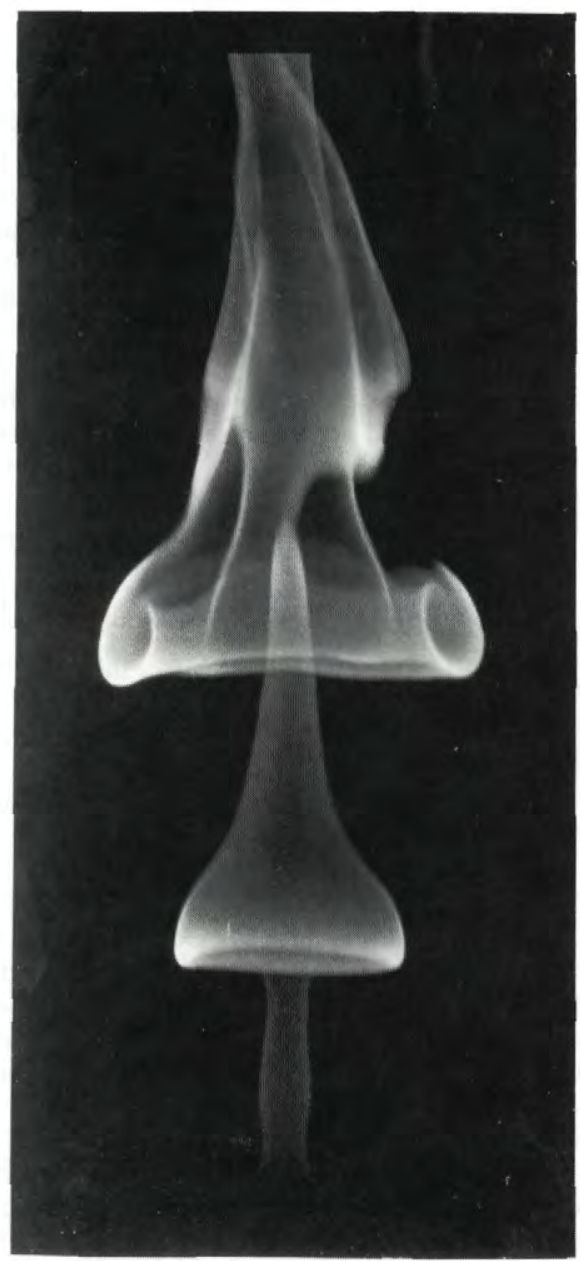

FIG. 8. Direct flow visualization of a round laminar jet diffusion flame axially forced at a frequency of $12 \mathrm{~Hz}$.

duce an azimuthal instability in the ring. The ring instability quickly saturates and the ring breaks up through a process of reconnection and pinching off of closed flame cells (closed vortex loops). Note that the evolution of this pattern is similar to the mode 3 found in the cold jet flow. Modes 2 and 4 were never observed experimentally for the combustion cases.

\section{Conclusions}

We have presented experimental evidence of the existence of a large variety of entrainment patterns resulting from different modes of induction of the jet's vorticity. Through a set of well controlled experiments, we have shown that resulting from the

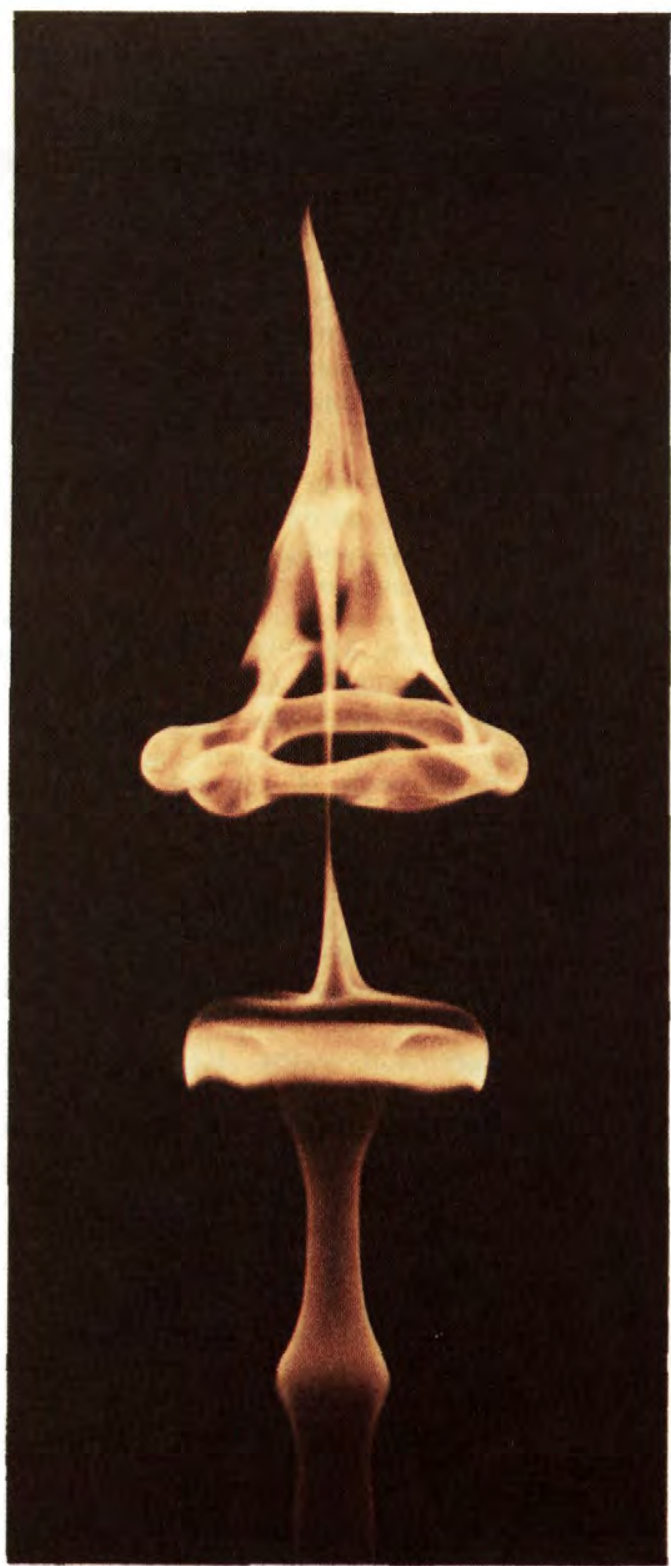

FIG. 9. Same case as in Figure 8. Nozzle with five lobes.

combined effect of streamwise and azimuthal forcing, the instability of the vortex rings formed in the jet results in the formation of closed vortex loops that under their own induction are propelled sideways away from the jet's axis.

For the case of corrugated nozzles, we have shown the existence of several three-dimensional modes all of which involve the formation of a symmetric 
pattern of close vortex loops. The observed threedimensional vortex patterns were interpreted in terms of the instability of two arrays of co-axial vortex rings of opposite sign. Depending on the relative strength between these co-axial vortex rings and on the size of their cores, we have shown that through a process of reconnection, closed vortex loops may form from either or both arrays of coaxial vortex rings, thus resulting in chunks of jet fluid ejecting sideways. Strongly forced jet diffusion flames are shown to exhibit the formation of some of the vortex patterns qualitatively similar to the cold flow.

\section{REFERENCES}

1. Mrchalke, A. and Freymunth, P.: The instability and the formation of Vortices in a free shear layer, AGARD, Conf. Proc. no. 4, paper 2. (1966).

2. WILLE, R.: Growth of velocity fluctuations leading to turbulence in a free shear layer. Heramn Fottinger Inst,, Berlin, AFOSR Tech. Rep. Contract AF 61(052)-412 (1963).

3. Becker, H. A. and Massaro, T. A.: J. Fluid Mech. 31, 435, 8 (1968).

4. Beavers, G. S. and Wilson, T. A.: J. Fluid Mech. 44, 97 70, 801 (1970).

5. Crow, S. and Champagne, F. M.: J. Fluid Mech. 48, 547 (1971).

6. Cohen, J. and Wygnanski, I.: J. Fluid Mech. Part 1. 176, 191 (1987).

7. Browand, F. K. and Laufer, J.: Proc. 4th Biennial Symp. Turbulence in Liquids, University of Missouri-Rolla, Princeton, New Jersey: Science Press, p. 33.3, (1975).

8. Yule, A. J.: J. Fluid Mech. 89, 413 (1978).

9. Dimotakis, P. E., Miake-Lye, R. C. and Pa. Pantoniou, D. A.: Phys. Fluids 26, 3185 (1983).

10. Tso, J. and Hussain, F.: J. Fluid Mech. 203, 425, (1989).

11. Mungal, M. G. and Hollingsworth, D. K.: Phys. Fluids A. 1, 1615 (1989).

12. Widnall, S. D. and Suldivan, J. P.: Proc. Roy. Soc. A, 332, 335 (1973).
13. Widnall, S. D. Bliss, D. B. And Tsai, C-Y.: J. Fluid Mech. 66, 35 (1974).

14. Maxworthy, T.: J. Fluid Mech. 51, 15 (1972).

15. Maxworthy, T.: J. Fluid Mech. 81, 465 (1977).

16. Martin, J. E., Meibukg, E. and Lasheras, J. C.: Three-dimensional evolution of axisymmetric jets: a comparison between computations and experiments. IUTAM Symposium on Separated Flows and Jets. July 9-13, Novosibirsk, USSR. (1990).

17. Meiburc, E., Lasheras, J. C. and Martin, J. E.: Experimental and numerical analysis of the three-dimensional cvolution of an axisymmetric jet. Turbulent Shear Flows 7 (F. Durst Ed.) Springer-Verlag (1989).

18. Martin, J. E. and Meiburg, E.: Three-dimensional evolution of an axisymmetric jet: a numerical investigation. J. Fluid Mech., 230, 271, (1991).

19. Lasheras, J. C. And Chol H.: J. Fluid Mech. 189,53 (1988).

20. Ashurst, W. T. and Meiburg, E.: J. Fluid Mech. 189, 87-98 (1988).

21. Mfiburg, E. and Lasheras, J. C.: J. Fluid Mech. 190, 1, (1988).

22. Lasheras, J. C. And Meiburg, E.: Phys of Fluids A. 2,3, 371 (1990).

23. Lecuona, A., Rodriguez, P. and Lasheras, J. C.: Three-Dimensional Structure of Strongly Forced Jets Diffusion Flames: Flow Visualization Studies. Combustion Flow Diagnostics (Durao, Whitelaw and Witze Ed.) Kluwer Academic Publishers, (Dordrecht, Holland).

24. Lasheras, J. C., Lecouna, A., MakTin, J. E., Mriblikg, E. and Rodriguez, P.: Three-dimensional Vorticity Patterns in Forced, Co-flowing Jets. J. Fluid Mech. in preparation, 1992.

25. Monkewitz, P. A., Lehmann B., Barsikow, B. And Bechent, D.: Phys. Fluids A. 1, 446 (1989).

26. Monkewitz, P. A., BeChent, D., Barsikow, B. and Leimanin B.: J. Fluid Mech. 213, 611, (1990).

27. Sreenivasan, K. R., Racihu, S. and Kylf, D.: Experiments in Fluids, 7, 309 (1989).

28. Subarao, E. R.: Ph.D. Thesis. Stanford University, (1987).

29. Liepmann, D.: Ph. D, Thesis. University of California, San Diego, (1990). 